\title{
MESA DE COORDENADAS CARTESIANAS (X,Y) PARA LA PERFORACIÓN DE MATERIALES POR MEDIO DE UN MICROCONTROLADOR 8051 DE INTEL
}

\author{
AUTOR: \\ Omar Yesid Flórez Prada \\ Estudiante de $\mathrm{X}$ semestre de ingeniería electrónica. \\ omar fl@control-automatico.net.
}

\section{RESUMEN}

En nuestro medio estamos rodeados de una serie de sistemas electrónicos que efectúan operaciones automáticas de acuerdo con una serie de parámetros programados anteriormente por el operario.

El presente trabajo muestra el prototipo de una mesa de dos coordenadas (plano cartesiano $(X, Y)$ ), la cual utiliza un sistema de desarrollo basado en el microcontrolador 8051 de INTEL (R) (sistema de computo), realizando la función de sistema de control enviando las respectivas ordenes, para ubicar la herramienta en los diferentes puntos del área de trabajo de la mesa, los puntos son programados con anterioridad por el operario, interactuando con el teclado. Para realizar los desplazamientos de la mesa $(X, Y)$, se utilizan dispositivos actuadores encargados de efectuar un movimiento lineal que desplaza la herramienta a la distancia determinada.

\section{PALABRAS CLAVES}

Microcontrolador, sistema de desarrollo, motores paso a paso, lenguaje ensamblador.

\section{INTRODUCION}

Los procesos de fabricación pueden clasificarse, según el grado de automatización y sofisticación de los sistemas de control, en niveles que van desde la producción manual hasta el máximo paradigma de la Manufactura Integrada por Computador (CIM), con las llamadas fabricas de luces apagadas. En estas, el operario que conocemos desaparece para dar paso a las máquinas, que se encargan totalmente de la producción. El hombre sólo realiza las operaciones de programación y de supervisión.

Se realizó un programa (software) en lenguaje ensamblador para un microcontrolador, controlando el giro del motor paso a paso para el desplazamiento de la coordenada $X$ y el giro del motor paso a paso para el desplazamiento de la coordenada $\mathrm{Y}$, encargados de ubicar la herramienta para la perforación del material en los puntos específicos.

La herramienta de perforación es manipulada por el operario por medio de teclado; la cual se desplaza hasta el punto $(X, Y)$ en el que se desea la perforación, utilizando la tecla " $H$ " se almacena esta coordenada, luego se desplaza la herramienta hasta una nueva coordenada $(X, Y)$ y se prosigue a su almacenamiento, así sucesivamente hasta haber indicado todas las perforaciones que se desean realizar.

Como el operador indica aleatoriamente cada posición, el programa entrega a la mesa $(X, Y)$ cada uno de los desplazamientos que deben realizar los motores paso a paso, de acuerdo con la información obtenida en la tabla de coordenadas, de este modo las perforaciones se llevarán ha cabo en una forma consecutiva.

Este tipo de control se ha realizado implementando computadoras personales con más capacidad de memoria y procesadores con grandes velocidades, el software utilizado es un lenguaje de alto nivel (orientado objetos) en el se realiza un dibujo en dos o tres dimensiones con un ambiente familiar, este se compila y envía los respectivos pulsos de control por un puerto de salida conectado con la mesa $(X, Y)$.

Una de las ventajas del proyecto comparado con las tecnologías existentes en el mercado, es su sistema de con-trol económico, menor requerimiento de memoria solo $4 \mathrm{~K}$ Bytes y la realización de tareas repetitivas, para su aplicación en la perforación en serie de material.

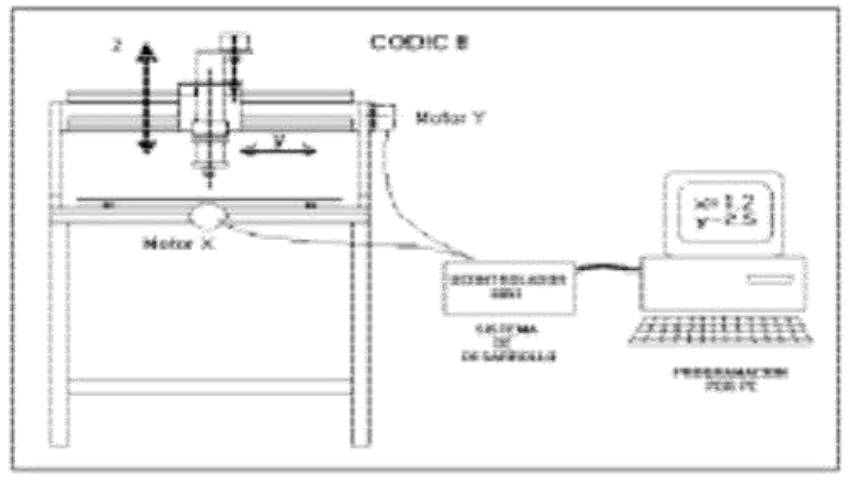




\section{APLICACION}

El propósito de realizar el proyecto es su implementación en diversos campos; como podemos ver el programa realizado es estándar y los cambios para determinada aplicación está dada por la selección del tipo de herramienta de corte y el tipo de material a trabajar (mármol, madera, yeso, hierro, baquelita, etc.).

Se puede implementar para perforar las baquelitas en la fabricación de circuitos impresos.

\section{DISEÑO DEL HARDWARE}

El sistema físico esta compuesto por los siguientes elementos:

\section{DIAGRAMA EN BLOQUES DEL HARDWARE}

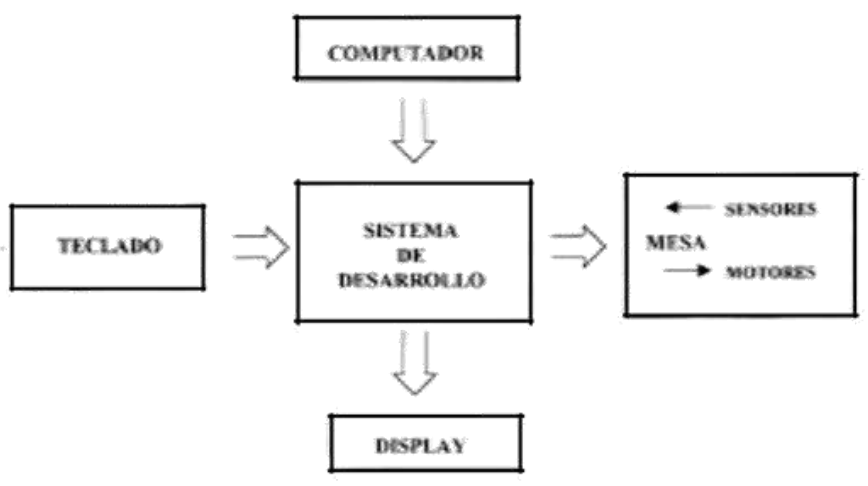

\section{COMPUTADOR}

Se emplea una computadora como herramienta de edición, utilizando el software MLED (R) creado por Microdiseño Ltda. (R). permite la escritura del programa en lenguaje ensamblador, terminado el programa se prosigue al envío por medio del puerto serial (COM 1) al sistema de desarrollo.

Las ventajas es la versatilidad que ofrece el computador en la búsqueda de errores, corregirlos e introducir en cualquier parte del programa una línea de instrucción o insertar un segmento de instrucciones.

\section{SISTEMA DE DESARROLLO}

El sistema tiene una arquitectura que dispone de blo-ques funcionales los cuales son:

CPU, es un microcontrolador 8051 de Intel que posibi-lita nuevas aplicaciones que se pueden llevar a cabo con la mínima utilización de componentes.

MEMORIA DE PROGRAMA EXTERNA (PROM), conformada por $64 \mathrm{~K}$ bytes, en ella se encuentra almacenado el programa monitor o sistema operativo (MOS51) del sistema de desarrollo.

MEMORIA DE DATOS EXTERNA (RAM), es posible direccionar hasta $64 \mathrm{~K}$ bytes, en esta se puede realizar tan-to la escritura como la lectura de datos en esta memoria.

MEMORIA COMBINADA RAM/ROM, esta segmentada en espacios utilizados por el sistema de desarrollo, en uno de esos segmentos de memoria se almacena el códi-go de programa, esta direccionada desde $9200 \mathrm{H}$ hasta 957FH (896 bytes) y tiene otro segmento de memoria libre para usuario, la cual almacena la tabla de coorde-nadas y su dirección es 9F00H hasta $9 F 7 F H$ (128 bytes).

PUERTOS, se programó el controlador de puertos 8255 (PPI) de la siguiente manera: el puerto $A$ de salida, en este se conecta la etapa de potencia para manejar el motor $X$ y el puerto $B$ como salida, conectando la etapa de potencia para manejar el motor $\mathrm{Y}$,

Por otra parte se utiliza el puerto P1 del microcontrolador para la entrada de las señales provenientes de los sensores, este puerto es direccionable bit a bit.

\section{TECLADO}

Su función es el ingreso de datos para editar el programa directamente en el sistema de desarrollo; En el programa permite la interfaz entre el sistema de desarrollo y el operario, permitiendo guiar la herramienta hasta 
los puntos donde se quiere perforar e ingresar estas coordenadas. Se configuro las teclas de la siguiente manera:

«D», movimiento a la derecha del eje $X$.

«A», movimiento a la izquierda del eje $X$.

$« W »$, movimiento hacia arriba el eje $Y$.

«X», movimiento hacia abajo el eje $\mathrm{Y}$.

«H», almacenamiento del respectivo punto $(X, Y)$ en el que se encuentra la herramienta.

«P», Con esta tecla se da inicio al recorrido automático de los diferentes puntos $(X, Y)$ anteriormente grabados, esta tarea se puede realizar repetitivamente.

\section{DISPLAY}

Consiste en una pantalla de cristal liquido (LCD), que muestra mensajes predefinidos en la programación del microcontrolador, para nuestro programa se visualizan dos mensajes:

Primero, se muestra el mensaje "PROGRAMACION», este indica al operario que el sistema se encuentra en modo de programación y tiene la posibilidad de ingresar las diferentes coordenadas moviendo la herramienta al respectivo punto $(X, Y)$ y almacenarlo.

Segundo, en el instante que se oprime la tecla «P» se muestra el mensaje «AUTOMATICO», la plataforma re-corre los puntos almacenados autónomamente.

\section{INTERFAZ DE POTENCIA}

Se utilizaron 8 transistores en configuración darlington (TIP 122), 4 transistores para cada uno de los respectivos motores, estos transistores tiene características como: diodo interno en antiparalelo para contracorriente, amplificación de corriente en factor de 1000 y corriente máxima en colector 8 amperios.

\section{MOTORES}

Un motor de paso es básicamente un motor cuya operación consiste en movimientos angulares discretos de magnitudes esencialmente uniformes en lugar de rotación continua. Este tipo de motor puede ser fácilmente controlado por circuitos electrónicos los cuales convierten pulsos eléctricos en movimientos mecánicos proporcionales. Cada fracción de revolución del motor es hecha por una serie de pasos discretos idénticos. El diseño del motor de paso usualmente provee rotación en el sentido de las manecillas del reloj así como en contra. Lo que hace al motor de paso idealmente conveniente para muchas aplicaciones de posición y de control. El hecho que los motores de paso puedan ser fácilmente controlados por pulsos eléctricos permite el uso de señales digitales para controlar el movimiento mecánico o la posición; además, el torque relativamente grande asociado con cada movimiento permite al motor de paso reemplazar aparatos como embragues y frenos, haciendo más seguros los sistemas.

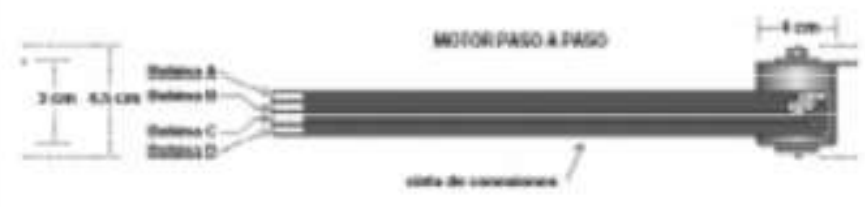

En el proyecto se utilizaron dos motores, uno para cada eje de coordenada con las siguientes características:

Motor unipolar, con $8^{\Omega}$ de impedancia por bobina. Angulo de giro por paso es de 1.8‥ Corriente suministrada $800 \mathrm{~mA}$ amperios. Voltaje de polarización es de 12 voltios.

\section{SENSORES}

Los sensores se utilizaron, para la entrada de señales al sistema de desarrollo, que indican la ubicación de la herramienta en la coordenada de inicio (0.0), a partir de este punto de referencia se inicia el conteo de cada una de las posiciones.

En el eje $X$ se implementó un sensor óptico, conformado por un emisor de luz infrarroja y un fototransistor como receptor, él entrega una señal en el momento que detecta el rayo luz infrarroja, indicando que el eje $X$ se encuentra en el punto $\mathrm{X}=0$.

En el eje $Y$ se implementó un micro switch, entregando una señal en el momento, que este eje se encuentra en el punto de origen. 


\section{MESA}

Por ser un prototipo, se construyo a escala pequeña, entregando las siguientes características: Resolución muy baja, esta característica es dada por los motores y el sistema de tracción, debe ser lo más baja para obtener una buena precisión, área de trabajo pequeña lo cual limita las medidas del material a trabajar (6 $\mathrm{cm}$ en el eje $X$ y $6 \mathrm{~cm}$ en el eje $Y$ )

\section{DISEÑO DEL SOFTWARE}

La metodología que se utilizó en el proceso secuencial para la realización del software tuvo en cuenta las características del proceso, buscando siempre su desarrollo real, para la aplicación coherente de los objetivos planteados. Los subprogramas se enuncian a continuación en un orden lógico:

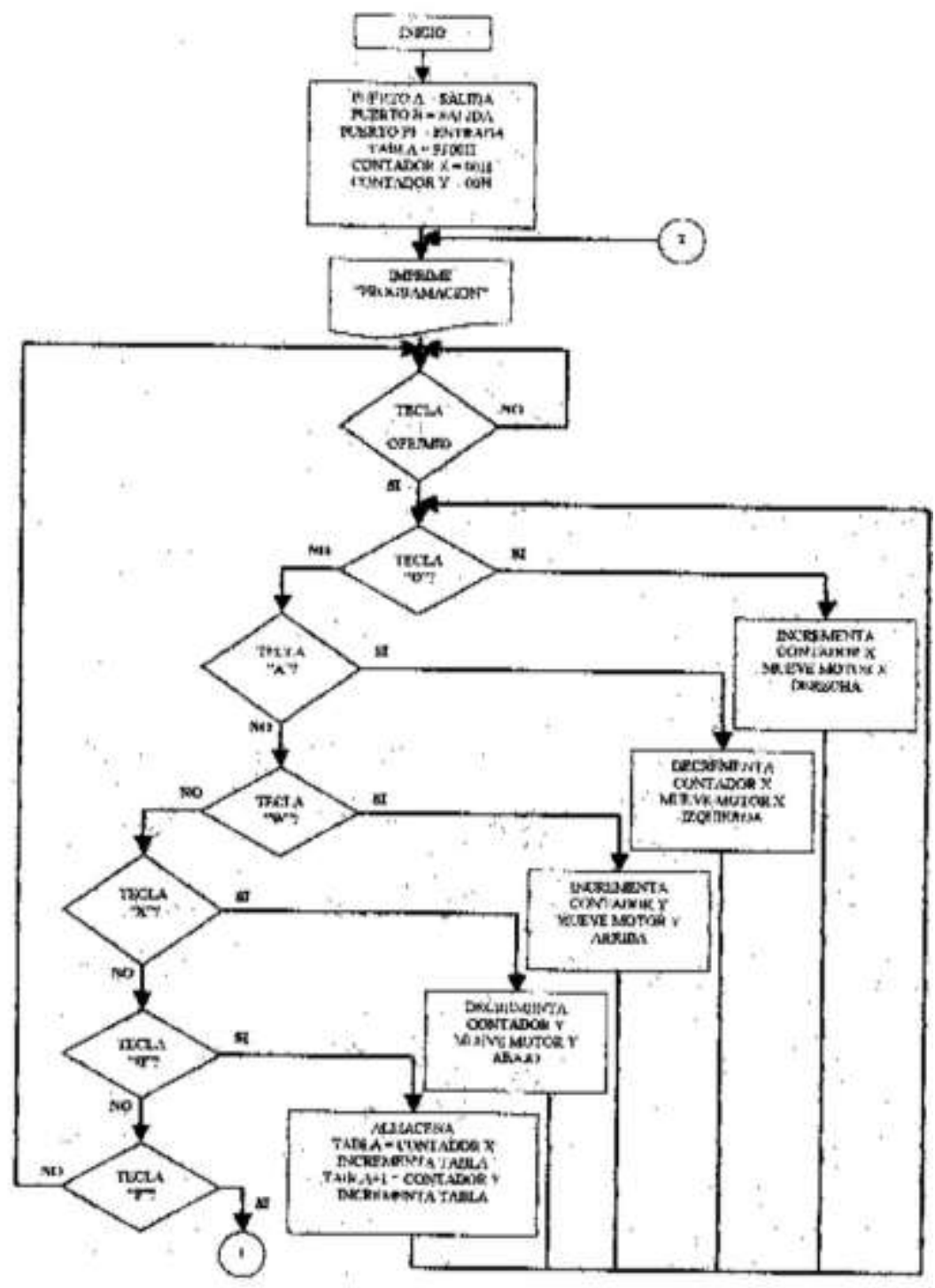

1.Subprograma para el movimien-to de la plataforma ubicando la herramienta en la respectiva coordenada por medio de las siguientes teclas: «D», mueve hacia la derecha el motor $\mathrm{X}$; «A», mueve hacia la izquierda el motor $\mathrm{X}$; «W», mueve hacia la derecha el motor $Y$; « $X$ » mueve hacia la izquierda el motor $Y$. Cada vez que se oprime una tecla, el movimiento equivalente es de $1.8^{\circ}$ en cada uno de los motores.

2.Subprograma que por medio de la tecla «H», almacena la coordenada respectiva en un sector de la memoria (segmento de datos) direccionado como $9 \mathrm{~F} 00 \mathrm{H}$, formando la tabla de coordenadas.

3.Subprograma que utiliza la tecla «P», para dar orden de recorrer automáticamente todos los puntos. Tiene la función de direccionar la tabla, tomar una coordenada y por medio de este dato, enviar las respectivas señales al puerto de salida en este se conecta la etapa de potencia que maneja los motores paso a paso de la mesa, para que se realice el desplazamiento automático de cada uno de los ejes y así sucesivamente hasta encontrar el fin de la tabla, OFFH indica que se ha terminado de ubicar la herramienta en todos los puntos programados por el operario.

4.Programa que evalúa las señales enviadas por sensores de fin de carrera, ubicados en la coordenada origen de la mesa, por medio de estas señales el programa debe tomar decisiones auxiliares. 


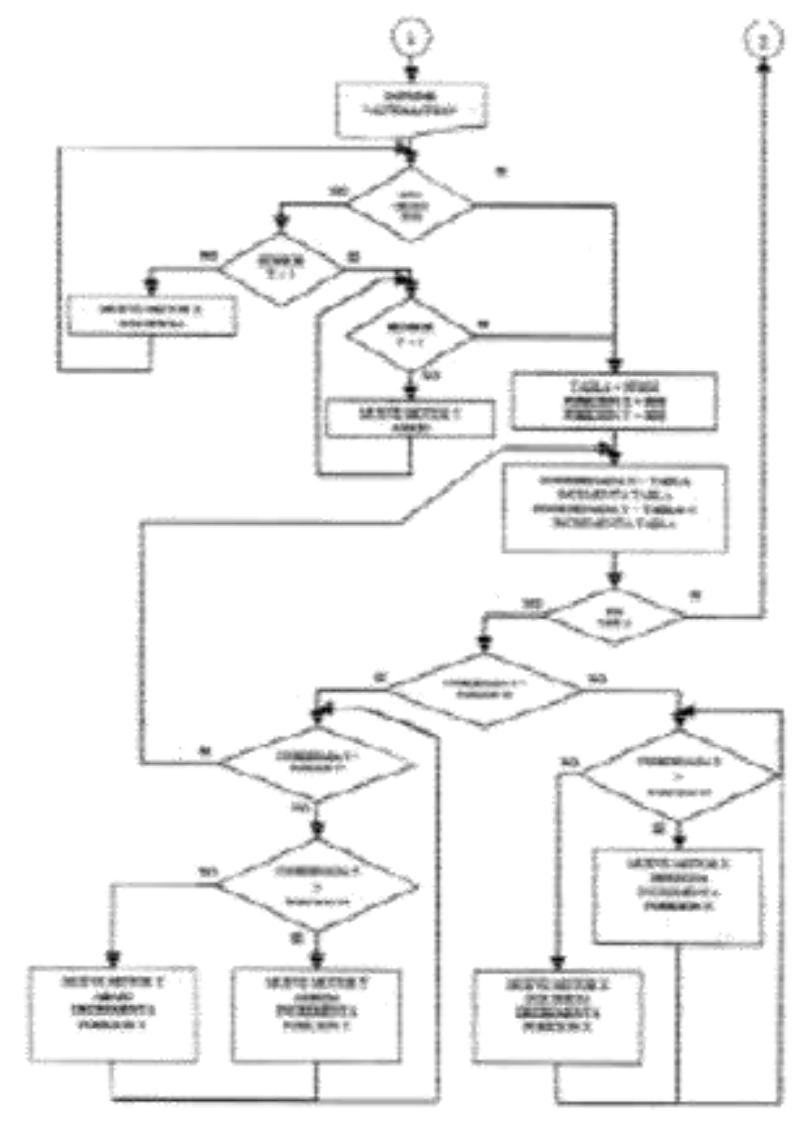

CONCLUSIONES

Una de las limitaciones, son las distancias máximas de cada eje, se ampliara el área de trabajo en la siguiente etapa del proyecto, con la construcción de una plataforma a gran escala. El prototipo que se ha desarrollado reproduce los movimientos del eje $X$ y del eje $Y$ con una velocidad optima y alta precisión, para la siguiente etapa del proyecto se realizará el acoplamiento del motor que realizará los movimientos de la herramienta para el perforamiento (control del eje Z).
El acoplamiento y adaptación de sensores de fin de carrera ubicados en los extremos superiores de cada eje son necesarios para evitar el desbordamiento de los contadores de posición y evitar que ocurra un choque de la plataforma al final de recorrido.

\section{BIBLIOGRAFIA}

ANGULO, José y ANGULO, Ignacio. Microcontroladores PIC. Madrid: McGraw-Hill,1999. 295p.

HORESTEIN, Mark N. Microelectrónica: Circuitos y Dispositivos. México: Prentice Hall, 1995. 126p.
MICRODISEÑO. Microlab-SI, Manual de Usuario. Medellín: s.n.,1995

\section{Sistema de Desarrollo} Portá-til para Microcontroladores de las Familias 80C51. Medellín: s.n., 1995, 102 p. +12 apéndices
OGATA, Katsuhiko. Sistemas de Control en Tiempo Discreto. México: Prentice Hall, 1996. 745p.

SAVAN, Jr; RODEN, Martín y CARPENTER, Gordon. California: Edison Wesley, 1992. 880p. 
